\title{
基于正交切削理论的熔石英高温本构参数的 逆向识别
}

\author{
潘鹏飞, 宋华伟, 任国旗, 杨佐辉, 肖峻峰, 陈肖, 许剑锋 ${ }^{*}$ \\ 华中科技大学机械科学与工程学院, 国家数字制造设备与技术重点实验室, 武汉 430074 \\ * E-mail: jfxu@hust.edu.cn \\ 收稿日期: 2020-02-26; 接受日期: 2020-06-19; 网络版发表日期: 2020-08-14 \\ 国家自然科学基金(批准号: 51627807, 51705172, 51905195)资助项目
}

摘要 激光辅助加工(laser-assisted machining, LAM) 是一种新型的热机械加工技术, 它为熔石英玻璃等硬脆材料 的高效、低成本的优质加工提供了一种新的途径. 但LAM工艺通常涉及大应变、大应变率和高温, 这些变量在 实验中是难以观测的. 有限元切削模拟是预测切削过程切削机理的一种有效手段, 而准确的材料本构模型是进行 精确仿真的关键. 为了获得更准确的模拟结果，本文提出了一种基于正交切削理论的材料本构关系的构建方法， 以弥补熔石英本构模型的缺失. 根据Oxley切削理论, 建立了主剪切区应力、应变、应变率和温度等参数的场分 布数学模型. 搭建了正交切削实验平台, 并根据实验结果计算出等效流动应力, 然后通过遗传算法(genetic algorithm, GA) 确定了熔石英的高温本构方程并应用于模拟中, 最后通过实验验证了切削仿真模型. 结果表明, 仿真能 较好地反映实际切削实验中的切削力和切屑形貌, 这说明了本构参数的准确性和 GA识别方法的可靠性.

关键词熔石英玻璃, 本构模型, 遗传算法, 正交切削, Johnson-Cook模型

\section{1 引言}

熔石英玻璃是一种主要由硅、氧元素组成的非晶 态固体，具有强度高、抗热震、耐腐蚀、耐高温和光 学特性优良等物化特性，因此被广泛应用于航空航 天、半导体、光电子、医药和化工等领域 ${ }^{[1]}$. 但熔石 英玻璃脆性高、断裂韧性低、强度和弹性极限非常接 近等特点使其很难通过常规加工方式高效地加工．激 光辅助加工(laser-assisted machining, LAM) 是一种新 型的热机械加工技术 ${ }^{[2]}$, 其原理是在切削前利用激光
束局部辐照提高材料待加工区的温度，软化被加工的 表层材料，同时增强材料的塑性流动特性，从而实现 难加工材料的塑性去除. 此外, LAM方法还可以显著 降低刀具磨损, 提高工件表面加工质量. 因此, LAM技 术在熔石英等硬脆材料的加工方面具有广阔的应用 前景 ${ }^{[3]}$.

切削过程中产生的切削力、应力和剪切区温度将 对刀具-工件界面产生强烈的耦合作用, 并影响着加工 表面质量和刀具寿命. 然而, 通过实验方法观测应力、 应变、温度的变化需要较高的设备成本, 且结果的准

引用格式: 潘鹏飞, 宋华伟, 任国旗, 等. 基于正交切削理论的熔石英高温本构参数的逆向识别. 中国科学: 技术科学, 2020, 50: 1426-1436 Pan P F, Song H W, Ren G Q, et al. Reverse identification of high-temperature constitutive parameters of fused silica based on orthogonal cutting theory (in Chinese). Sci Sin Tech, 2020, 50: 1426-1436, doi: 10.1360/SST-2020-0057 
确性难以保障. 有限元方法(finite element method, FEM)使用计算机辅助科学来模拟切削过程, 它不仅可 以准确地预测切削过程中的各种切削变量, 而且可用 于分析切削机理. 然而, 仿真模型的可靠性依赖于材 料本构关系的精准建立，对于伴随着大应变、高温和 大应变率的LAM过程而言, 本构方程是决定模型能否 准确描述热机械耦合效应特征的关键.

目前, 国内外学者对熔石英的切削加工方面的研 究较少, 这在一定程度上导致了熔石英的动态力学性 能参数的缺乏，进而限制了熔石英材料在某些关键应 用中的持续发展. Bonora等人 ${ }^{[4]}$ 利用逆向标定技术确 定了熔石英材料的Johnson-Holmquist动态本构模型参 数, 进行了泰勒圆柱体冲击实验和落锤实验, 并采用多 目标优化方法对实验数据进行了本构参数识别, 通过 数值预测和实验结果对比验证了本构参数的有效性. 然而，传统的冲击实验获得的材料流动特性很难准确 地描述材料在大应变、大应变速率切削过程中的热塑 性变形的问题. Ritcher等人 ${ }^{[5]}$ 为了在激光修复仿真模 型中更直观地反映石英玻璃在高温下的蠕变和应力松 弛行为, 通过单轴压缩实验数据建立了石英玻璃的 Maxwell模型，用以描述其应力应变关系. 李润强 ${ }^{[6]}$ 对 熔石英玻璃的动态黏弹性测量实验数据进行数值拟 合，从而确定了熔石英玻璃的广义Maxwell模型参数, 随后建立了修复后的石英玻璃表面残余应力的有限元 模型, 获得了残余应力的分布规律, 并通过实验验证了 有限元模型的正确性. 然而, Maxwell模型处理高应变 率、大变形问题的局限性，使其很难被应用于描述动 态切削过程.

由此可见，选择合适的本构模型对于熔石英在高 温下本构关系建立尤为重要. 在常用的材料本构模型 中, Johnson-Cook (J-C) 模型是一个与温度、应变率有 关的经验型耦合模型，适用于描述熔石英在LAM中的 热机械耦合过程.

一般地，材料本构模型的建立的方法主要有三种: 有限元法、霍普金森压杆(split Hopkinson pressure bar, SHPB)实验法和切削实验法. 通过国内外学者在本构 方程建立方面的大量研究发现, 相对于单一的SHPB 实验法, 采用Oxley塑性剪切理论 ${ }^{[7]}$ 与寻优算法结合的 方式建立的本构方程能较好地描述材料的加工硬化行 为. 例如, $P$ ang 等人 ${ }^{[8]}$ 通过切削实验逆向辨识了 AISI1045的J-C本构参数. 首先采用修正的Oxley经典
的平行剪切带模型计算了剪切区的应力、应变、应变 率和温度; 然后利用遗传算法逆向辨识本构参数; 最后 通过仿真模型与实验进行对比验证, 结果表明, 实验与 仿真的一致性说明了该辨识方法的有效性.

综上，本文旨在解决熔石英高温动态力学特性参 数的缺失问题，提出了一种基于正交切削理论的材料 本构关系的构建方法，并搭建了正交切削实验平台. 利用Oxley基本切削理论, 通过切削实验的切削力和切 屑厚度结果来计算材料的主剪切区的应变、应力、应 变率和温度等物理量. 依据SHPB实验获得初始本构参 数范围, 以理论值与实验值之间的最小误差为目标函 数，通过遗传算法的全局搜索能力获得了最优本构参 数. 建立了基于所获得的 J-C本构参数的有限元切削模 型，并通过切削实验验证了逆向识别方法的可行性和 本构模型的准确性.

\section{2 剪切区模型}

前期的研究 ${ }^{[9]}$ 表明，熔石英在激光的辅助加热下 表现出一定的塑性变形能力. 当工件表面被激光辐射 充分加热时，材料将被软化并表现出与金属切削类似 的塑性特性. 为了进一步探讨高温下熔石英的切削机 理，本节基于Oxley ${ }^{[7]}$ 塑性剪切理论和Tounsi等人 ${ }^{[10]}$ 不 等分剪切带理论, 进一步推导了剪切带中应力、应 变、应变速率和温度的场分布.

图1描绘了正交切削的不等分剪切模型. 其中, $t_{1}$ 是切削厚度, $t_{2}$ 为切屑厚度, $V$ 是切削速度, $V_{c}$ 是切屑流 速, $V_{s}$ 是剪切速度, $h$ 是剪切区厚度. 该模型的建立基于 以下假设：

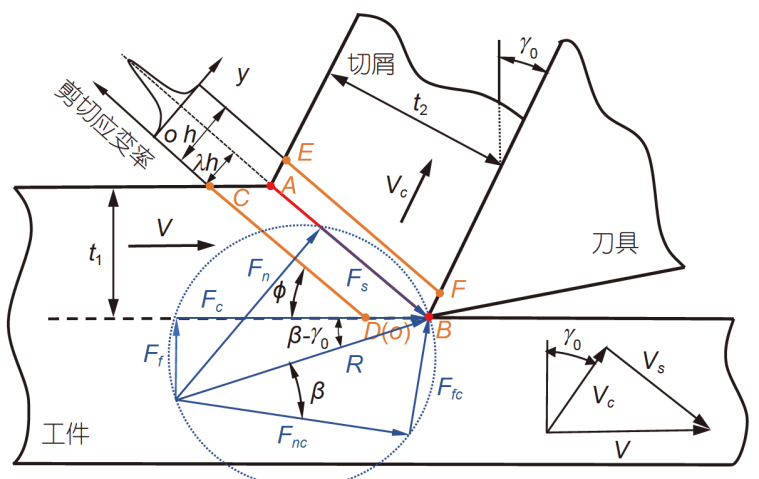

图 1 (网络版彩图)不等分剪切区模型

Figure 1 (Color online) Unequal shear zone model. 
(1) 剪切滑移是第一变形区切屑形成的主要机制;

(2) 工件材料均匀、各向同性, 满足Johnson-Cook 热黏塑性本构方程;

（3）切削过程稳定, 各截面上的材料形变沿切削宽 度方向一致, 满足平面应变假设 ${ }^{[11]}$;

(4) 主剪切区厚度不随时间变化;

(5) 忽略了刀尖钝圆半径对主剪切区的影响.

在该模型中, 主剪切面 $A B$ 将剪切带分成两个厚度 不相等的部分. 待加工材料经过始剪切滑移线 $C D$ 进入 剪切区; 然后在刀具的挤压和摩擦作用下而发生剪切 滑移; 最终通过终剪切滑移线 $E F$ 后, 切屑被去除. 从主 剪切面到初始剪切线的距离是整个剪切带厚度的 $\lambda$ 倍, 不等分因子 $\lambda<1$.

\section{1 变形控制方程}

根据理论计算发现，由切削实验测量获得的切屑 厚度可以计算出切削模型中的剪切角 $\phi$ :

$\phi=\arctan \frac{r_{c} \cos \gamma_{0}}{1-r_{c} \sin \gamma_{0}}$,

其中, $r_{c}=t_{1} / t_{2}$ 为变形系数.

通过力学分析，可以将切削合力 $R$ 分解为垂直于 切削方向的进给力 $F_{f}$ 和平行于切削方向的主切削力 $F_{c}$. 则剪切力 $F_{s}$ 和法向力 $F_{n}$ 可表示为

$F_{s}=F_{c} \cos \phi-F_{f} \sin \phi$,

$F_{n}=F_{c} \sin \phi+F_{f} \cos \phi$.

假设主剪切面上的剪切应力 $\tau_{A B}$ 是均匀分布的, 并 且剪切力 $F_{S}$ 与 $\tau_{A B}$ 成比例, 则

$\tau_{A B}=\frac{F_{s}}{A_{\mathrm{s}}}=\frac{\sin \phi}{a t_{1}} F_{s}$,

其中, $A_{s}$ 是主剪切平面的面积, $a$ 为切削宽度.

结合式(2)和式(4)可得

$\tau_{A B}=\frac{\sin \phi}{a t_{1}}\left(F_{c} \cos \varphi-F_{f} \sin \phi\right)$.

如图2所示, 对剪切区边界的运动进行速度分解, $V_{0 t}$ 和 $V_{h t}$ 是切向速度分量, $V_{0 n}$ 和 $V_{h n}$ 是法向速度分量.

根据运动关系, 速度分量如下:

$\left\{\begin{array}{l}V_{0 t}=-V \cos \phi \\ V_{0 n}=V \sin \phi \\ V_{h t}=V \sin \phi \tan \left(\phi-\gamma_{0}\right), \\ V_{h n}=V_{c} \cos \left(\phi-\gamma_{0}\right) .\end{array}\right.$

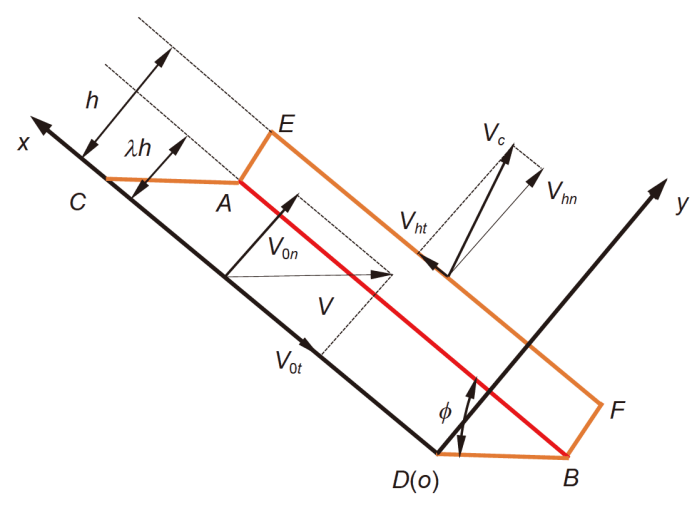

图 2 (网络版彩图)剪切区边界的速度模型

Figure 2 (Color online) Velocity decomposition of shear zone boundaries.

根据塑性不可压缩条件 ${ }^{[12]}$ ，即通过剪切区的法向 速度是恒定的, 则切屑速度可由公式(6)获得:

$V_{c}=V \sin \phi / \cos \left(\phi-\gamma_{0}\right)$.

剪切应变率 $\gamma$ 是剪切应变的随体导数 ${ }^{[13]}$, 则

$\dot{\gamma}=\frac{\mathrm{d} \gamma}{\mathrm{d} t}=\frac{\partial \gamma}{\partial t}+\frac{\partial \gamma}{\partial y} \frac{\partial y}{\partial t}=V \sin \phi \frac{\mathrm{d} \gamma}{\mathrm{d} y}$.

根据Oxley理论, 可假设剪切应变率在剪切变形区 呈分段非线性分布 ${ }^{[14]}$. 即剪切应变率 $\gamma_{A B}$ 在主剪切面上 最大, 在剪切区的边界上近似为零. 因此, 剪切区中的 应变率场可以表示为

$\dot{\gamma}= \begin{cases}\dot{\gamma}_{A B} \cdot(y / \lambda h)^{q}, & 0 \leq y \leq \lambda h, \\ \dot{\gamma}_{A B} \cdot((h-y) /(h-\lambda h))^{q}, & \lambda h<y \leq h,\end{cases}$

其中, $q$ 是不确定性系数, 它描述了剪切区中切向速度 的非一致性. 通过Shi等人 ${ }^{[15]}$ 的研究, 当 $q=3$ 时, 剪切区 的应力分布可以很好地被描述.

考虑到切向速度 $V_{x}$ 的边界条件 $V_{0 t}$ 和 $V_{h t}$, 由相容条 件 ${ }^{[16]}$, 对剪切应变率积分可得速度场

$v_{x}= \begin{cases}\frac{\dot{\gamma}_{A B} \cdot y^{q+1}}{(q+1)(\lambda h)^{q}}+V_{0 t}, & 0 \leq y \leq \lambda h, \\ -\frac{\dot{\gamma}_{A B} \cdot(h-y)^{q+1}}{(q+1)(h-\lambda h)^{q}}+V_{h t}, & \lambda h<y \leq h .\end{cases}$

由式(10)可知，切向速度的符号在剪切区边界 $C D$ 和 $E F$ 处发生了变化, 而主剪切平面的切向速度为零:

$\left.v_{x}\right|_{y=\lambda h}=0$. 
联立方程(10)可得

$\dot{\gamma}_{A B}=\frac{(q+1) V \cos \gamma_{0}}{h \cos \left(\phi-\gamma_{0}\right)}$,

$\lambda=\frac{\cos \phi \cos \left(\phi-\gamma_{0}\right)}{\cos \gamma_{0}}$.

结合式(8)和式(9), 考虑到始剪切线 $C D$ 处的应变 等于零, 则对方程(9)积分可得

$$
\begin{aligned}
& \gamma= \\
& \begin{cases}\frac{\dot{\gamma}_{A B} \cdot y^{q+1}}{(q+1) V \sin \phi \cdot(\lambda h)^{q}}, & 0 \leq y \leq \lambda h, \\
-\frac{\dot{\gamma}_{A B}(h-y)^{q+1}}{(q+1) V \sin \phi \cdot(h-\lambda h)^{q}}+\frac{\lambda}{\tan \phi}, & \lambda h<y \leq h,\end{cases}
\end{aligned}
$$

则主剪切面应变为

$\gamma_{A B}=\frac{k \cos \gamma_{0}}{\sin \phi \cos \left(\phi-\gamma_{0}\right)}=\frac{1}{\tan \phi}$.

\section{2 温度控制方程}

切削过程中通常将剪切区边界以内视为绝热区 域, 传热可忽略不计. 根据Oxley切削理论和Boothroyd 温度模型 ${ }^{[17]}$, 剪切平面 $A B$ 上的平均切削温度为

$T_{A B}=T_{0}+\Delta T_{A B}=T_{0}+\frac{(1-\xi) F_{s} \cos \gamma_{0}}{\rho c t_{1} a \cos \left(\phi-\gamma_{0}\right)}$,

其中, $T_{0}$ 为工件的初始温度, $\Delta T_{A B}$ 是温升, $\zeta$ 是切削热传 入工件的比例, 它是由无量纲热常数 $R_{T}$ 决定的, $\rho$ 是材 料的密度, $c$ 为比热容.

$\xi=\left\{\begin{array}{cc}0.5-0.35 \lg \left(R_{T} \tan \phi\right), & 0.04 \leq R_{T} \tan \phi \leq 10.0, \\ 0.3-0.15 \lg \left(R_{T} \tan \phi\right), & R_{T} \tan \phi>10.0,\end{array}\right.$

$R_{T}=\frac{\rho c V t_{1}}{K}$,

其中, $K$ 为材料的导热系数.

\section{3 本构参数的逆向辨识}

Johnson-Cook模型是一种与应变率和温度紧密相 关的经验型黏塑性模型，适用于描述大多数塑性材料 在大应变、高应变率和变化环境温度下的力学性能, 它具有形式简单和物理参数意义明确的特点. 因此, 熔 石英的高温热机械耦合特性可以用 $J-C$ 本构方程 ${ }^{[18]}$ 来 描述:
$\bar{\sigma}_{A B}=\left(A+B \bar{\varepsilon}_{A B}^{n}\right)\left[1+C \ln \left(\frac{\dot{\bar{\varepsilon}}_{A B}}{\dot{\bar{\varepsilon}}_{0}}\right)\right]\left[1-\left(\frac{T_{A B}-T_{0}}{T_{m}-T_{0}}\right)^{m}\right.$,

式中, $\bar{\sigma}_{A B}$ 为等效流动应力, $A$ 为材料室温时的屈服强 度, $B$ 为加工硬化模量, $C$ 为应变率常数, $n$ 为硬化指数, $m$ 为温度软化系数, $\bar{\varepsilon}_{A B}$ 为等效塑性应变, $\dot{\bar{\varepsilon}}_{A B}$ 为等效塑 性应变率, 取 $1 \mathrm{~s}^{-1}, \dot{\bar{\varepsilon}}_{0}$ 为应变率参考值, $T_{0}$ 为环境温度, 取 $25^{\circ} \mathrm{C}, T_{m}$ 为材料的熔点温度.

\section{1 本构识别方法}

图3为本构参数的识别过程. 首先, 基于剪切区理 论, 通过正交切削实验获得的切削力和切屑厚度等参 数以及初始切削条件(切削速度、切削宽度、切削厚 度、刀具前角)计算等效流动应力. 等效流动应力与主 剪切面剪应力的数学关系如下:

$\bar{\sigma}_{\text {exp }}=\sqrt{3} \tau_{A B}, \quad \bar{\varepsilon}_{A B}=\frac{\gamma_{A B}}{\sqrt{3}}, \quad \dot{\bar{\varepsilon}}_{A B}=\frac{\dot{\gamma}_{A B}}{\sqrt{3}}$.

然后, 采用分离式霍普金森压杆(SHPB)实验研究

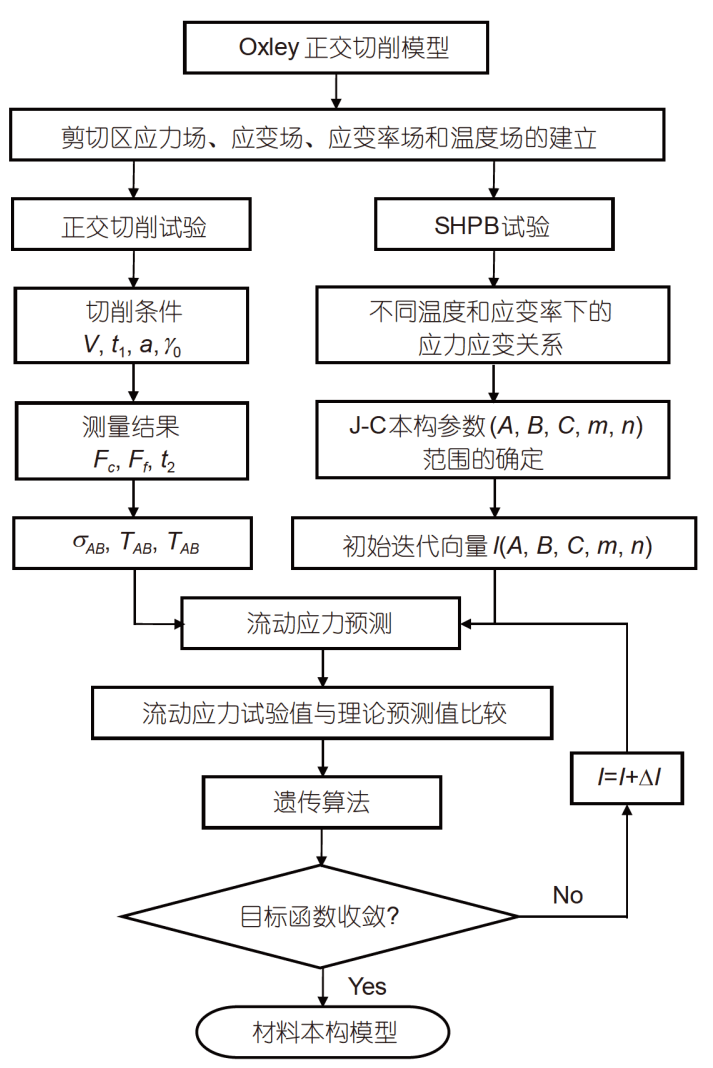

图 3 本构参数识别流程

Figure 3 Identification process of constitutive parameters. 
了高应变速率下的塑性流动应力，得到了熔石英的本 构参数范围. SHPB实验装置如图4所示，在传统的 SHPB装置中增加了高温加热装置和温度控制装置. 加热装置能达到的最高温度为 $1000^{\circ} \mathrm{C}$. SHPB实验的 应变率分别为 800,1200 和 $1800 \mathrm{~s}^{-1}$, 实验温度分别为 $20^{\circ} \mathrm{C}, 500^{\circ} \mathrm{C}$ 和 $1000^{\circ} \mathrm{C}$. 每组实验重复进行三次. 根据 一维应力波理论, 通过实验数据计算了实样的应力、 应变和应变率. 然后，根据最小二乘法拟合得到初始 的J-C本构参数, 并确定参数范围, 如表 1 所示.

最后, 为了得到准确可靠的流动应力参数, 以预测 的流动应力 $\bar{\sigma}_{A B}$ 与实验应力 $\bar{\sigma}_{\mathrm{exp}}$ 之间的平均相对误差作 为遗传算法的适应度函数(式(21)), 并在表 1 所示的参 数范围内进行全局寻优.

$(A, B, C, m, n)=\min \left\{\frac{1}{N} \sum_{i=1}^{N}\left(\frac{\bar{\sigma}_{A B}}{\bar{\sigma}_{\mathrm{exp}}}-1\right)^{2}\right\}$.

\section{2 正交切削实验}

通过坐标变换，正交切削过程可以等效为旋转切 削过程. 本研究中的LAM切削实验是在通用 CNC机床 上进行的, 实验装置如图5所示.

实验装置由切削系统和激光辅助加热系统组成. 热源采用 $\mathrm{CO}_{2}$ 脉冲激光器( $\left.\mathrm{SR} 10 \mathrm{i} \mathrm{ROFIN}\right)$, 其波长为
$10.6 \mu \mathrm{m}$ ，脉冲频率为 $0 \sim 100 \mathrm{kHz}$ ，脉冲能量为 $5 \sim 100 \mathrm{~mJ}$, 最大脉冲占空比为 $60 \% . \mathrm{CO}_{2}$ 激光束经由反 射镜、激光扩束器和聚焦透镜组成的可调光学系统传 送至切削系统中, 在工件表面形成直径为 $3 \mathrm{~mm}$ 的圆形 光斑. 激光束-刀具的轴向间距可调, 以确保工件在被 切削前预热. 尺寸为 $\phi 10 \mathrm{~mm} \times 60 \mathrm{~mm}$ 的工件被装夹在 机床主轴上, 并依靠主轴的旋转和进给来实现正交切 削. 激光束和刀具呈 $180^{\circ}$ 分置于工件两侧, 用安装于 标准刀架上的多晶金刚石(PCD)刀具进行了熔石英的 LAM实验. 刀具的型号参数列于表 2 .

设计了两因子四水平的正交切削实验. 切削速度 $V$ 选择为 $11.3,15.1,18.8$ 和 $22.6 \mathrm{~m} / \mathrm{min}$ 四个水平. 切削 厚度 $t_{1}$ 选择为 $0.01,0.015,0.02$ 和 $0.025 \mathrm{~mm} / \mathrm{r}$ 四个水平. 确定切削宽度为 $0.1 \mathrm{~mm}$, 实验采用的激光功率为 $80 \mathrm{~W}$ (对应占空比 $42 \%$ ).

实验中，采用安装于刀具下方的测力仪(Kistler 9257A) 实时测量三个切削力分量, 放大器采样频率为

$1 \mathrm{kHz}$. 实验后，采用扫描电子显微镜 (SEM, model: Helios, NanoLab, G3, CX)观测切屑形貌及切屑厚度. 由于切屑易碎且尺寸极小, 很难通过制样法精确测量 其截面厚度, 因此通过SEM观察了切屑的厚度. 为减 小实验误差，每组实验重复进行了三次，以平均切削 力和切屑厚度作为实验结果. 正交切削实验结果如表 3所示.

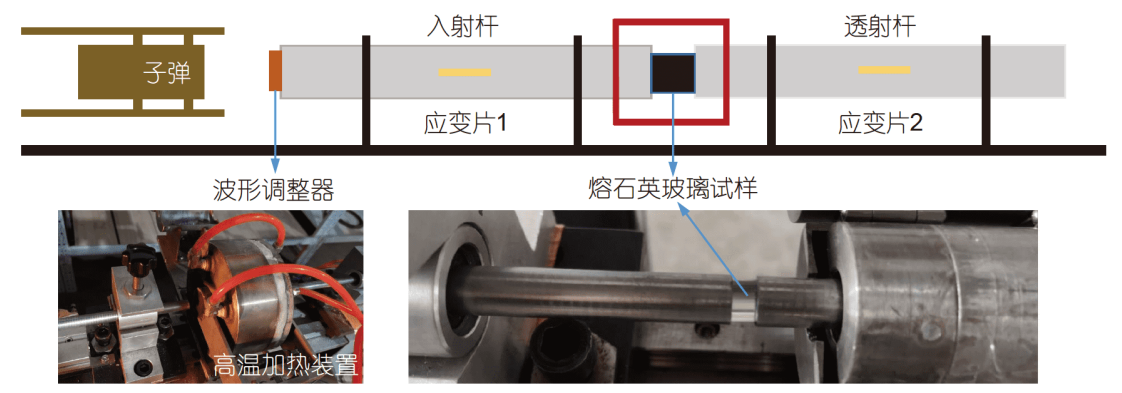

图 4 (网络版彩图) SHPB实验装置

Figure 4 ( Color online) The setup of SHPB experiment.

表 1 熔石英Johnson-Cook参数范围

Table 1 Johnson-Cook parameters range of the fused silica

\begin{tabular}{cccccc}
\hline 参数 & $A(\mathrm{MPa})$ & $B(\mathrm{MPa})$ & $C$ & $m$ & $n$ \\
\hline 初始值 & 300 & 1197 & 0.4543 & 1.163 & 0.0043 \\
范围 & $100 \sim 500$ & $800 \sim 1400$ & $0.001 \sim 0.5$ & $0.01 \sim 2$ & $0.0001 \sim 4$ \\
\hline
\end{tabular}




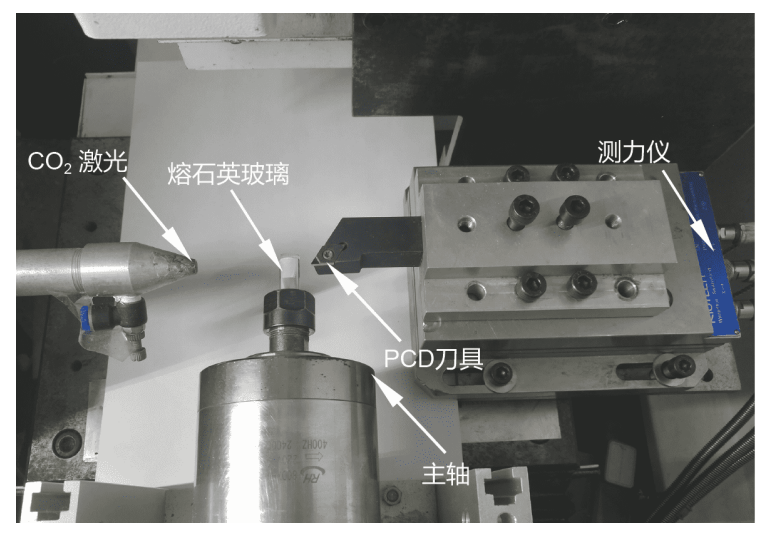

图 5 (网络版彩图)切削实验装置

Figure 5 (Color online) The setup of cutting experiment.

\section{3 遗传算法}

遗传算法 $(\mathrm{GA})$ 是为全局最优设计的随机搜索算 法, 它模仿自然选择的生物进化过程 ${ }^{[19]}$. 本研究通过 遗传算法寻找误差函数(式(21))的最小值, 从而获得最 优的流动应力参数. 迭代向量 $\boldsymbol{l}(A, B, C, m, n)$ 中的流动 应力参数设为单个染色体基因，不同的基因组合形成 不同的个体，而一定数目的个体构成一个种群，个体 的数量是种群的大小.
表 2 刀具参数表

Table 2 Tool summary

\begin{tabular}{cc}
\hline 参数 & 描述 \\
\hline 刀具型号 & DCMT11T304 \\
刀具形状 & 菱形顶角 $55^{\circ}$ \\
刀具前角 & $7^{\circ}$ \\
刀具后角 & $7^{\circ}$ \\
切削刃长 & $11 \mathrm{~mm}$ \\
刀具厚度 & $3.97 \mathrm{~mm}$ \\
刀尖半径 & $0.4 \mathrm{~mm}$ \\
刀杆型号 & SDJCR2020k11 \\
主偏角 & $93^{\circ}$ \\
\hline
\end{tabular}

遗传算法中的关键参数包括种群大小、变异率、 交叉率和最大迭代次数等, 这些参数对计算结果和计 算时间都有着重要影响:

(1) 种群规模一般为20 200, 种群规模过小会使最 优参数求解困难, 反之, 则收玫时间变长;

(2) 变异率是基因在染色体中变异的概率, 通常取 为 $0.0001 \sim 0.1$, 变异率太小会导致新的基因结构出现概 率极低, 反之, 变异率太大, GA可能转变为随机搜索

表 3 正交切削实验与结果

Table 3 Conditions and results of orthogonal cutting experi-ments

\begin{tabular}{|c|c|c|c|c|c|}
\hline 序号 & 切削速率 $(\mathrm{m} / \mathrm{min})$ & 切削厚度 $(\mathrm{mm} / \mathrm{r})$ & 主切削力 (N) & 进给力 $(\mathrm{N})$ & 切屑厚度 $(\mathrm{mm})$ \\
\hline 1 & 11.3 & 0.01 & 7.32 & 4.39 & 0.0190 \\
\hline 2 & 11.3 & 0.015 & 9.24 & 5.61 & 0.0281 \\
\hline 3 & 11.3 & 0.02 & 10.83 & 6.28 & 0.0322 \\
\hline 4 & 11.3 & 0.025 & 11.96 & 7.42 & 0.0379 \\
\hline 5 & 15.1 & 0.01 & 6.67 & 4.36 & 0.0182 \\
\hline 6 & 15.1 & 0.015 & 8.93 & 5.67 & 0.0276 \\
\hline 7 & 15.1 & 0.02 & 9.49 & 5.98 & 0.0308 \\
\hline 8 & 15.1 & 0.025 & 10.38 & 6.73 & 0.0353 \\
\hline 9 & 18.8 & 0.01 & 6.30 & 3.89 & 0.0177 \\
\hline 10 & 18.8 & 0.015 & 8.07 & 5.42 & 0.0256 \\
\hline 11 & 18.8 & 0.02 & 8.79 & 5.47 & 0.0294 \\
\hline 12 & 18.8 & 0.025 & 9.65 & 6.18 & 0.0328 \\
\hline 13 & 22.6 & 0.01 & 6.27 & 3.64 & 0.0158 \\
\hline 14 & 22.6 & 0.015 & 7.37 & 4.39 & 0.0226 \\
\hline 15 & 22.6 & 0.02 & 8.68 & 5.38 & 0.0288 \\
\hline 16 & 22.6 & 0.025 & 9.74 & 5.99 & 0.0305 \\
\hline
\end{tabular}


方法;

（3）交叉率表示染色体某段发生交叉的概率, 通常 在0.6 0.95之间. 交叉率太小会导致搜索停滞, 太大则 可能会破坏高适应度值的结构.

结合研究内容综合考虑, GA中的四个参数分别设 为 $100,0.01,0.65$ 和 300 , 算法容差为 $10^{-6}$.

图6所示为遗传算法的流程. 首先, 从表 2 所列初始 参数范围内随机生成初始种群; 然后, 使用适应度函数 (式(21))来评估个体, 以更小的累积误差来衡量个体被 选用的概率, 概率较大的个体有更大的可能性将其特 征传递给下一代; 最后，次代经由当代染色体的选 择、交叉和变异而产生. 由于次代都是从当代中更好 的适应度染色体中获得，所以适应度会逐步提高. 经 过一定次数的迭代后，适应度将稳定收玫，迭代即 结束.

如图7所示, 经过 200 次迭代, 适应度函数基本稳定

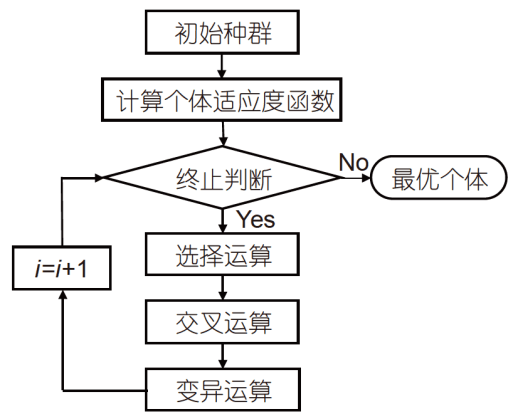

图 6 GA算法流程

Figure 6 Genetic algorithm process.

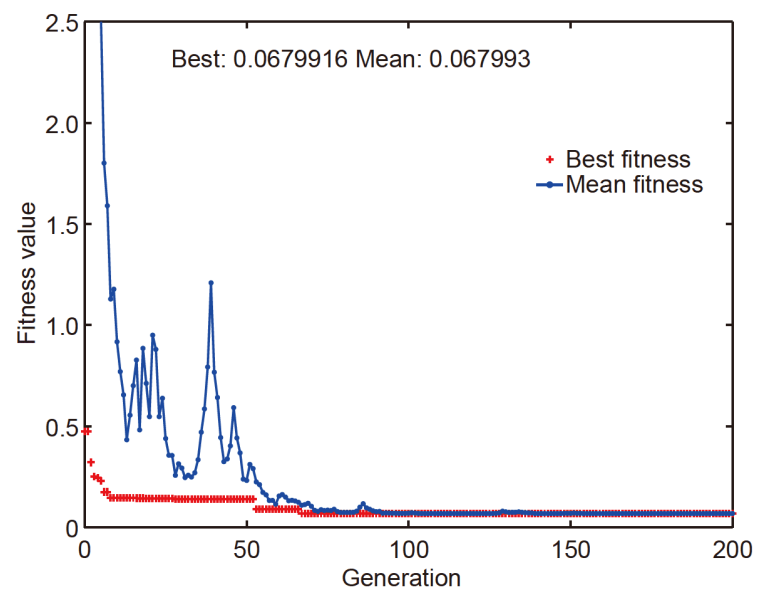

图 7 (网络版彩图)适应度值随种群代数的变化

Figure 7 (Color online) The variety in fitness values with the population algebra.
并收玫. 获得的适应度函数最小值为 0.06799 , 对应的 流动应力参数为: $A=207.6, B=1035.2, C=0.021$, $m=0.103, n=0.6$. 因此, 逆向识别 ${ }^{[20]}$ 获得的本构方程的 表达式为

$$
\begin{aligned}
\bar{\sigma}_{A B}= & \left(207.6+1035.2 \bar{\varepsilon}^{0.6}\right)\left[1+0.021 \ln \left(\frac{\dot{\bar{\varepsilon}}}{\overline{\bar{\varepsilon}_{0}}}\right)\right] \\
& \times\left[1-\left(\frac{T-T_{0}}{T_{m}-T_{0}}\right)^{0.103}\right] .
\end{aligned}
$$

\section{4 模拟结果和讨论}

为了验证本构参数的可靠性，建立了基于辨识获 得的本构方程的有限元切削模型.

\section{1 切削模型}

采用LS-DYNA有限元软件对熔石英的激光辅助 正交切削过程进行了模拟仿真，模型的几何原理图如 图8所示.

本节采用光滑粒子流体动力学 $(\mathrm{SPH})$ 与有限元方 法(FEM)耦合的方式建立了切削模型，简化了计算的 同时, 避免了在计算大动态变形中网格畸变的发生. 切削模型由工件和刀具两部分组成. 工件上部分, 即 切屑形成和大变形发生的区域, 由SPH粒子构成; 而下 部采用 FEM网格建模. 矩形工件的尺寸为 $100 \mu \mathrm{m} \times 50 \mu \mathrm{m} \times 20 \mu \mathrm{m}$. 刀具采用有限单元法建模, 刀 具前角为 $7^{\circ}$, 后角为 $7^{\circ}$. 模型中使用的参数列于表 4 .

在切削模型中，采用刀具相对于固定工件运动的 工况以模拟正交切削过程的相对运动. 模型的边界条 件和约束如图8所示. 通过定义沿 $X$ 轴负方向的恒定速

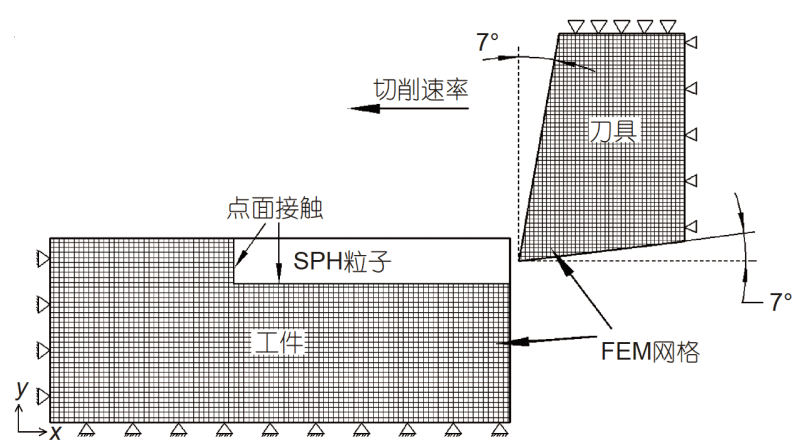

图 8 切削模型示意图

Figure 8 A schematic presentation of the cutting model. 
表 4 刀具和工件的物理参数

Table 4 Physical parameters of the workpiece and the tool

\begin{tabular}{cccc}
\hline 物理参数 & 单位 & 工件 & 刀具 \\
\hline 密度 $(\rho)$ & $\mathrm{g} / \mathrm{cm}^{3}$ & 2.21 & 3.5 \\
弹性模量 $(E)$ & $\mathrm{Gpa}$ & 21 & 850 \\
剪切模量 $(G)$ & $\mathrm{Gpa}$ & 30.98 & - \\
泊松比 $(\mu)$ & - & 0.17 & 0.07 \\
比热容 $\left(C_{\mathrm{p}}\right)$ & $\mathrm{J} /\left(\mathrm{kg}^{\circ} \mathrm{C}\right)$ & 1211 & 471 \\
热导率 $(k)$ & $\mathrm{W} /\left(\mathrm{m}^{\circ} \mathrm{C}\right)$ & 2.4 & - \\
熔点 $\left(T_{\mathrm{melt}}\right)$ & ${ }^{\circ} \mathrm{C}$ & 1730 & - \\
室温 $\left(T_{\text {room }}\right)$ & ${ }^{\circ} \mathrm{C}$ & 25 & 25 \\
\hline
\end{tabular}

度来模拟刀具的运动, 并限制刀具的其他自由度, 以防 止刀具偏移的s发生. 同时，工件下边界和左边界的所 有方向均被固定. 建立SPH与FEM间耦合的难点在于 定义粒子区域与网格区域之间的界面。本模型利用 LS-DYNA提供的节点-表面接触来实现两部分的耦合. 仿真模型的运动参数与实验保持一致.

结合测温实验和COMSOL温度场仿真发现，切削 区域小体积范围内的温度变化很小, 而几何模型的最 大切削厚度不超过 $50 \mu \mathrm{m}$, 因此可将模型中工件的温 度边界设置为均匀温度场, 由温度场仿真确定 $80 \mathrm{~W}$ 的 激光功率对应的切削区初始温度为 $1210^{\circ} \mathrm{C}$.

切削过程是非常复杂的，因为刀具和切屑之间的 界面始终处于高温和高压的环境下. 刀具表面的摩擦 应力代表着刀具-切屑界面的摩擦, 它与切削力、切削 温度和刀具应力有关，并主要依赖于工件材料、切削 速度、进给速率和切削厚度. 目前, 绝大多数的切削 模拟都采用了修正的库仑摩擦模型. 其摩擦系数描述 如下:

$f=\frac{F_{c} \tan \gamma_{0}+F_{f}}{F_{c}-F_{f} \tan \gamma_{0}}$.

本研究中, 利用正交切削实验获得的切削力分量 计算出平均摩擦系数为 0.47 . 此外, 模型所采用的本构 参数与 $\mathrm{GA}$ 优化结果一致, 并选择 $\mathrm{J}-\mathrm{C}$ 分离准则作为材 料失效准则.

\section{2 实验验证}

本节从切削力和切屑形貌两个方面对仿真模型和 切削实验结果(前六组)进行了比较分析. 模型中使用
的切削条件与各组实验条件一致.

主切削力和进给力分别对应于模型中 $X$ 和 $Y$ 方向 上的切削力分量. 仿真和实验中切削力分量的对比如 图9所示，可以发现，两种切削力分量的实验结果与仿 真结果相近, 但存在一定的偏差. 切削力和进给力的最 大偏差值分别为 $13.72 \%$ 和 $16.16 \%$ ，且预测值小于实验 值. 除了几何模型简化等一些客观因素外, 激光加热引 起的材料去除和工件温度场的简化均导致较小预测切 削力的产生. 此外, 在切削模型中未考虑后刀面摩擦和 刀具磨损，这也是误差产生的原因. 总之，除去客观因 素的影响，该模型可以在误差允许的范围内较准确地 预测切削过程中的切削力.

图10为第六组实验及其对应仿真的切屑形态. 采 用SEM观察了实验中收集切屑的微观形貌. 由图可知, 在仿真和实验中均存在大尺寸连续切屑和半连续切 屑. 这是因为高温增强了熔石英的塑性特性, 促进了连 续切屑的生产, 当切屑脱离高温区域时, 其表面温度降
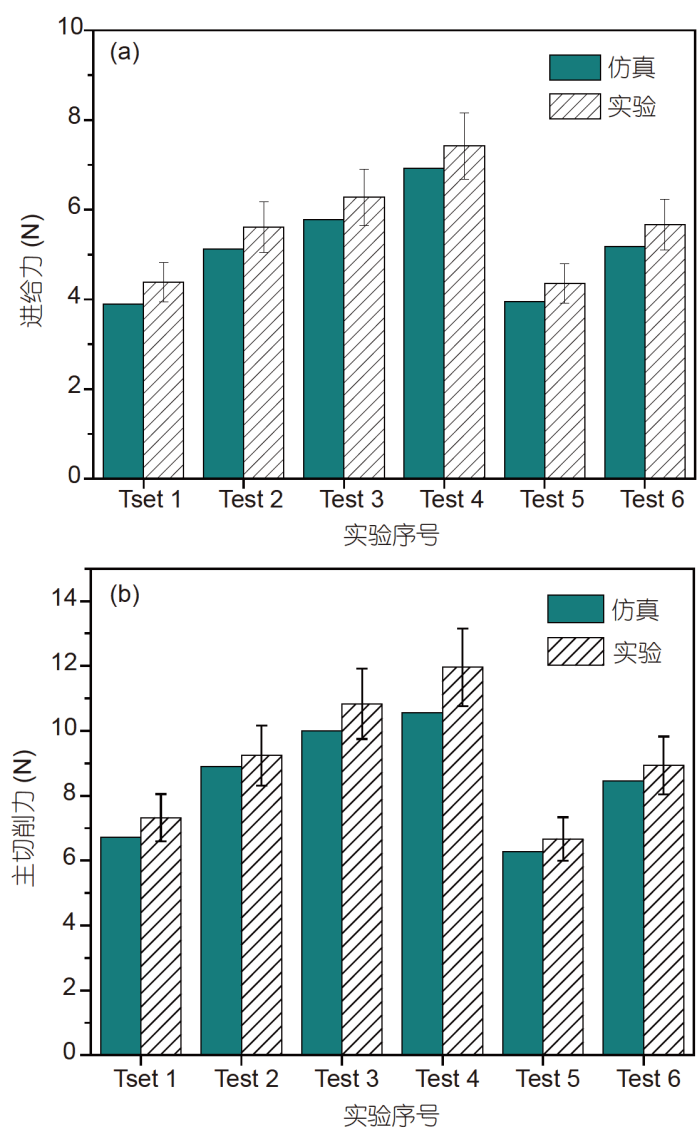

图 9 (网络版彩图)切削力验证实验结果

Figure 9 (Color online) Verification of cutting force. 

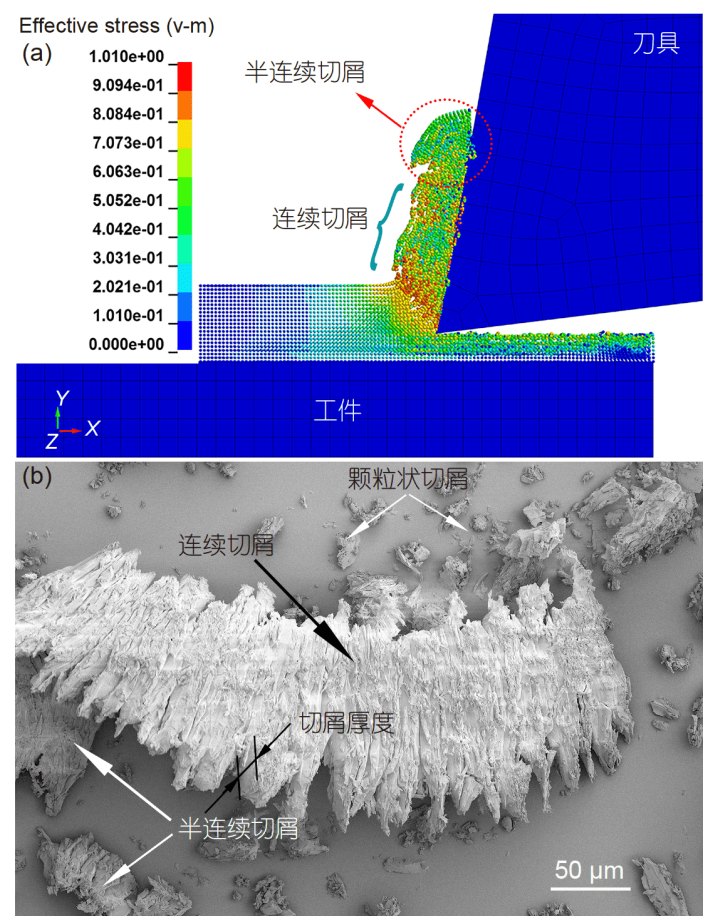

图 10 (网络版彩图) 切屑形态验证结果

Figure 10 (Color online) Verification of chip morphology.

低, 材料恢复了初始的硬度和脆性, 从而导致被去除材 料断裂形成半连续切屑. 同时, 部分半连续切屑不可避 免地是由连续切屑在收集过程中的断裂产生的. 图10(b) 中, 连续切屑周围存在颗粒状切屑, 这是由于切削过程
中激光加热的不均匀性导致局部材料软化不足，而材 料保持了较高的硬度和脆性，并通过刀具的挤压和摩 擦而得到颗粒状碎屑. 实验和模拟均可得到半连续和 连续的切屑，这表明材料的去除机理是脆性断裂和塑 性变形的混合模式.

切削力和切屑形貌在实验和模拟中的一致性说明 了 Johnson-Cook本构模型的准确性和可靠性，并证明 了基于遗传算法的本构参数辨识方法的可行性.

\section{5 结论}

本研究基于Oxley正交切削理论, 采用遗传算法识 别了熔石英的高温本构参数. 主要结论如下.

基于正交切削理论建立了剪切区应力场、应变 场、应变率场和温度场的数学模型; 进行了正交切削 实验，并根据切削力和切屑厚度测量结果计算了主剪 切面的流动应力.

通过高温SHPB实验确立了流动应力的初始参数 范围; 将流动应力在实验和理论的相对误差设为适应 度函数，采用遗传算法对熔融石英在高温下的本构参 数进行了逆向识别.

建立了三维正交切削模型，并将仿真结果与实验 结果进行比较, 结果表明, 仿真能较好地反映实际切 削实验中的切削力和切屑形貌，这说明了本构参数的 准确性和GA识别方法的可靠性.

致谢＼cjkstart切屑形貌及尺寸的观测实验在华中科技大学先进制造与技术实验中心的朱岩工程师的协助下顺利完成, 在此深 表谢忱.

\section{参考文献}

1 Dai W, Xiang X, Jiang Y, et al. Surface evolution and laser damage resistance of $\mathrm{CO}_{2}$ laser irradiated area of fused silica. Optics Lasers Eng, 2011, 49: 273-280

2 Samant A N, Dahotre N B. Laser machining of structural ceramics-A review. J Eur Ceramic Soc, 2009, 29: 969-993

3 Venkatesan K, Ramanujam R, Kuppan P. Laser assisted machining of difficult to cut materials: Research opportunities and future directions-A comprehensive review. Procedia Eng, 2014, 97: 1626-1636

4 Bonora N, Iannitti G, Ruggiero A, et al. Determination of Johnson-holmquist constitutive model parameters for fused silica. EPJ Web of Conferences, 2012, 26

5 Richter F, Hoffmann $\mathrm{H}$ J. Uniaxial compression of $\mathrm{SiO}_{2}$ glass cylinders: Analysis using stress-independent material coefficients. Mat-wiss u Werkstofftech, 2011, 42: 172-187

6 Li R Q. Study on the technology of laser repairing for cracks and stress relieving for the ground surface of fused silica (in Chinese). Dissertation 
for Master Degree. Jinan: Shandong University, 2017 [李润强. 石英玻璃表面的磨削裂纹激光修复和去应力工艺技术研究. 硕士学位论文. 济南: 山东大学, 2017]

7 Williams J A. Mechanics of Machining: An Analytical Approach to Assessing Machinability. Chichester: Halsted Press, 1989

8 Pang L, Kishawy H A. Modified primary shear zone analysis for identification of material mechanical behavior during machining process using genetic algorithm. J Manufacturing Sci Eng, 2012, 134: 041003

9 Song H, Dan J, Chen X, et al. Experimental investigation of machinability in laser-assisted machining of fused silica. Int J Adv Manuf Technol, 2018, 97: 267-278

10 Tounsi N, Vincenti J, Otho A, et al. From the basic mechanics of orthogonal metal cutting toward the identification of the constitutive equation. Int J Machine Tools Manufacture, 2002, 42: 1373-1383

11 Cui H L. Study on the cutting physical simulation of aluminum alloy 7050-T7451 based on material properties and tool-chip friction experiments (in Chinese). Dissertation for Master Degree. Chengdu: University of Electronic Science and Technology of China, 2013 [崔海龙. 基于材料特性 与刀屑摩擦实验的铝合金7050-T7451切削物理仿真研究. 硕士学位论文. 成都: 电子科技大学, 2013]

12 Huang X Z, Cao H, Zhang Y M, et al. Probabilistic analysis for cutting force in orthogonal cutting using Monte-Carlo method (in Chinese). J Northeastern Univ (Nat Sci), 2015, 36: 254-258 [黄贤振, 曹辉, 张义民, 等. 基于蒙特卡罗方法的直角切削切削力概率特性分析. 东北大学学 报(自然科学版), 2015, 36: 254-258]

13 Rees D. Basic Engineering Plasticity. Amsterdam: Elsevier, 2006

14 Li B, Wang X, Hu Y, et al. Analytical prediction of cutting forces in orthogonal cutting using unequal division shear-zone model. Int J Adv Manuf Technol, 2011, 54: 431-443

15 Shi B, Attia H, Tounsi N. Identification of material constitutive laws for machining - Part II: Generation of the constitutive data and validation of the constitutive law. J Manufacturing Sci Eng, 2010, 132: 051009

16 Li B L. Research on analytical prediction of cutting forces in stainless steel machining (in Chinese). Dissertation for Doctoral Degree. Wuhan: Huazhong University of Science and Technology, 2012 [李炳林. 不锈钢加工中切削力分析预测研究. 博士学位论文. 武汉: 华中科技大学, 2012]

17 Boothroyd G. Temperatures in orthogonal metal cutting. Arch P I Mech Eng, 1963, 177: 789-810

18 Bagci E. 3-D numerical analysis of orthogonal cutting process via mesh-free method. Int J Phys Sci, 2011, 6: 1267-1282

19 Wu G H, Wen Y, Yue M F, et al. Genetic algorithm and its application (in Chinese). Chin J Appl Mech, 1996, 13: 93-97 [武广号, 文毅, 乐美峰, 等. 遗传算法及其应用. 应用力学学报, 1996, 13: 93-97]

20 Pujana J, Arrazola P J, M'Saoubi R, et al. Analysis of the inverse identification of constitutive equations applied in orthogonal cutting process. Int J Machine Tools Manufacture, 2007, 47: 2153-2161 


\title{
Reverse identification of high-temperature constitutive parameters of fused silica based on orthogonal cutting theory
}

\author{
PAN PengFei, SONG HuaWei, REN GuoQi, YANG ZuoHui, XIAO JunFeng, \\ CHEN Xiao \& XU JianFeng
}

State Key Laboratory of Digital Manufacturing Equipment \& Technology, School of Mechanical Science and Engineering, Huazhong University of Science and Technology, Wuhan 430074, China

Laser-assisted machining (LAM) is a new type of thermomechanical processing technology that provides high-efficiency, low-cost, and high-quality processing of fused silica and other hard, brittle materials. However, the LAM process usually involves large strains, large strain rates, and high temperatures, which are difficult to observe in experiments. Finite element cutting simulations are effective for predicting the cutting mechanism of the cutting process, but an accurate material constitutive equation must be used for an accurate simulation. To obtain more accurate simulation results, this paper proposes a method for constructing the constitutive relationship of materials based on orthogonal cutting theory to compensate for the lack of a constitutive model for fused silica. According to the Oxley cutting theory, a mathematical model of the field distribution of parameters, such as stress, strain, strain rate, and temperature, in the main shear zone was established. An orthogonal cutting test platform was established, and the equivalent flow stress was calculated according to the test results. Then, a high-temperature constitutive equation for fused silica was determined by the genetic algorithm (GA) and applied to the simulation. Finally, the cutting simulation model was verified by experiments. The results showed that the simulation can better reflect the cutting force and chip morphology during an actual cutting test, which confirms the accuracy of the constitutive parameters and the reliability of the GA identification method.

fused silica, constitutive model, genetic algorithm, orthogonal cutting, johnson-cook model

doi: $10.1360 /$ SST-2020-0057 